\title{
Mulemba
}

Revista Angolana de Ciências Sociais

\section{Globalização e glocalização. 0 difícil diálogo entre o global e o local}

Globalisation and glocalisation. The difficult dialogue between the global and the local.

\section{Nelson Lourenço}

\section{OpenEdition \\ Journals}

\section{Edição electrónica}

URL: http://journals.openedition.org/mulemba/203

DOI: $10.4000 /$ mulemba.203

ISSN: 2520-0305

\section{Editora}

Edições Pedago

\section{Edição impressa}

Data de publição: 1 novembro 2014

Paginação: 17-31

ISSN: 2182-6471

\section{Refêrencia eletrónica}

Nelson Lourenço, «Globalização e glocalização. O difícil diálogo entre o global e o local», Mulemba

[Online], 4 (8) | 2014, posto online no dia 28 novembro 2016, consultado o 26 janeiro 2021. URL: http:// journals.openedition.org/mulemba/203 ; DOI: https://doi.org/10.4000/mulemba.203

Este documento foi criado de forma automática no dia 26 janeiro 2021.

Tous droits réservés 


\section{Globalização e glocalização. 0 difícil diálogo entre o global e o local}

Globalisation and glocalisation. The difficult dialogue between the global and the local.

Nelson Lourenço

\section{NOTA DO EDITOR}

Artigo solicitado ao Autor

Recepção do manuscrito: 12/12/2014

Aceite para publicação: 20/12/2014

\section{NOTA DO AUTOR}

Este artigo foi escrito no âmbito das actividades do programa de investigação que coordeno, sobre Globalização e Modernidade Reflexiva, no Instituto de Direito e Segurança da Universidade Nova de Lisboa (FD-UNL). As matérias aqui abordadas foram integradas nas aulas que ministrei em 2014 em Luanda no seminário Sociedade global, geopolítica e relações internacionais, do Curso de Doutoramento em Ciências Sociais e no Seminário Avaliação de Políticas Públicas, do Curso de Mestrado em Ciência Política e Administração Pública, ambos da Faculdade de Ciências Sociais (FCS) da Universidade Agostinho Neto (UAN). As interessantes discussões havidas com os alunos foram um estímulo à sua publicação, que ocorre aqui graças ao amável convite que me foi dirigido pelo Editor desta revista. 


\section{Introdução}

1 O vocábulo glocal é hoje de uso corrente.

2 Embora nem sempre seja fácil datar a origem dos conceitos, é correntemente aceite que a primeira referência ao termo glocalização aparece na década de 1980, sendo consagrada no The Oxford Dictionary of New Words, referindo-se ao processo de «telescoping global and local to make a blend». O conceito foi inicialmente construído a partir do vocábulo japonês dochakuka que originalmente se referia à adaptação das novas técnicas agrícolas às condições locais de produção. Rapidamente adoptado e vulgarizado no mundo dos negócios, o seu significado mais comum refere-se a um produto ou serviço concebido e distribuído globalmente mas adaptado aos hábitos e costumes locais.

3 A sua frequente utilização deve-se ao sucesso das políticas de marketing das grandes empresas multinacionais, particularmente das que se posicionam no mercado global, que adaptam os seus produtos e serviços aos contextos sociais e culturais em que os vendem, fazendopontes entre o globalismo e o localismo. Mas a sua preocupação centra-se no mercado e no cliente locais: fashion and customize, são as palavras-chave.

4 Os exemplos são muitos, tantos quanto a vulgarização do termo, aqui registam-se apenas três: a McDonalds que não vende hamburgers de vaca na Índia; a Whirlpool Corporation que adaptou as suas máquinas de lavar roupa aos longos saris usados pelas mulheres na Índia, passando a incorporar um espremedor especial para não estragar os tecidos; os motores de busca no mundo da $W W W$, como o Google e o Yahoo, têm versões adaptadas e contextualizadas de acordo com grandes áreas de clientes nacionais.

5 O objectivo deste artigo não é, no entanto, a análise das práticas de marketing no contexto da mundialização da economia, o que constituiria uma visão redutora do real significado dos termos glocal e glocalização, e da sua capacidade de fazerem salientar aspectos relevantes da problemática global versus local (ROBERTSON 1995). É sobre este quadro analítico que se desenvolve este artigo.

6 Um último comentário introdutório. As sociedades modernas têm vivido um processo de tão rápidas e intensas mudanças estruturais - que se tem vindo a acentuar, aliás, na última década - cujas consequências são dificilmente apreendidas pelo quadro de conceitos das ciências sociais, essencialmente construídos nos finais do século XIX e ao longo do século XX.

7 Os trabalhos de Ulrich Beck têm enfatizado esta necessidade de se construírem novos conceitos - «out of the existing conceptual arsenal, butin relation of empirical world» (BECK, SLATER e RITZER 2001: 263) - que facilitem uma acrescida apreensão das transformações que a sociedade moderna enfrenta. Esta é uma realidade que atravessa este artigo. Conceitos como global, regional e local, cultura e identidade, modernidade e modernização, exigem uma renovada e atenta leitura.

\section{Globalização e estruturação social}

8 A globalização, enquanto processo, na dimensão em que hoje a entendemos, ${ }^{1}$ representa algo de novo e com uma capacidade estruturadora da sociedade por vezes 
perturbante, simultaneamente desencadeadora de ameaças e proporcionadora de oportunidades à escala mundial, regional e local.

9 Embora os mecanismos e as etapas da evolução não sejam uniformes e apresentem traços e singularidades específicas dos vários contextos nacionais, a urbanização, a mundialização da economia e o desenvolvimento científico e tecnológico, as transformações estruturais dos sectores produtivos com a terciarização da economia e a diminuição da população activa na indústria, e particularmente, na agricultura, e o consequente e acentuado êxodo rural, são factores estruturais associados à globalização e à modernidade que emerge, assim, como uma realidade inerentemente globalizante (GIDDENS 1995; SKAPSKA 1997) embora não homogénea, como pertinentemente as ideias de construção de múltiplas modernidades (EISENSTADT 2007), de pluralização de modernidades (BECK, SLATER e RITZER 2001) e variadas modernidades (LIPOVETSKY 2011) explicitam.

10 A revolução científica e tecnológica, que acentuou os factores constitutivos da modernidade, impôs um dinamismo e um ritmo de mudança e de abrangência das transformações como nenhuma outra sociedade conheceu, afectando profundamente as práticas sociais e os modos de comportamento preexistentes (GIDDENS 1994: 14).

11 O desenvolvimento das tecnologias de comunicação e de informação resultantes da revolução digital, intensificaram os efeitos do processo de globalização mudando a natureza do tempo e do espaço, alterando a noção de distância, cruzando fronteiras e descontextualizando muitas das instituições e das práticas sociais. A globalização da modernidade emerge, assim, como um processo de compressão do tempo e de aniquilação do espaço, na definição de David Harvey (1989) a que se associa a internacionalização do capital, o consumismo e a construção de um mercado global. Refira-se que estas mudanças são também componentes essenciais no surgimento de novas ameaças e riscos, como uma nova criminalidade e de novas formas de violência, cujo sentido de localidade se integra na trama social e económica da globalização (LOURENÇO 2013b).

12 O recurso à noção de modernidade reflexiva, proposta por Anthony Giddens (1987) na sua teoria da estruturação social, permite integrar a análise da relação global-local no quadro do processo de globalização e de construção da modernidade. Opção justificada pelo papel crucial que a globalização desempenha na constituição da sociedade e dos processos sociais actuais e pela inegável capacidade explicativa do paradigma ${ }^{2}$ da globalização das mudanças sociais e culturais, sendo significativa a sua importância no quadro da moderna teoria social (FEATHERSTONE e LASH 1995).

13 Nas últimas duas décadas, isto é, na passagem do século XX para o século XXI, as transformações societais no quadro da modernidade não apenas se aceleraram como alteraram profundamente os quadros de referência: um novo tipo de capitalismo, um novo tipo de trabalho, um novo tipo de quotidiano, um novo tipo de Estado, um novo tipo de estrutura familiar, uma nova cultura.

14 Nos seus estudos sobre a globalização e modernidade Anthony Giddens refere-se a um novo período histórico que designa por modernidade tardia (GIDDENS 1994); retendo a aceleração das mudanças decorrentes da revolução do digital, Ulrich Beck fala em segunda modernidade (BECK e GRANDE 2010). Estes dois teóricos da globalização, referem-se essencialmente à passagem da sociedade industrial para a sociedade da informação, para a constituição de uma sociedade em rede, uma sociedade mais reflexiva e assente numa ordem pós-tradicional. Neste quadro, refiram-se as obras de 
Zygmunt Bauman em torno do seu conceito de modernidade líquida - isto é, da passagem de uma "hardware-focused modernity to a "light" and "liquid", software-based modernity» (BAUMAN 2000) - que constituem uma interessante, mesmo quando especulativa, leitura da incertitude e volatilidade das relações sociais e institucionais destes tempos.

\section{Globalização e glocalização}

15 É na década de 1980 que a globalização entra no discurso científico, assumindo um lugar proeminente para traduzir as mudanças sociais, económicas e culturais que a sociedade enfrentava. À frequente utilização correspondeu/correspondem um conjunto alargado e frequentemente diferenciado de definições salientando diferentes dimensões da realidade societal.

16 Perspectivada quer como processo (ROBERTSON 1995) quer como consequência (GIDDENS 1995), a avaliação dos impactes da globalização traduz uma inquietação visível quer no discurso científico quer, principalmente, nos discursos social e político que oscila, como diz Peter Beyer, entre uma «promessa utópica e uma ameaça distópica» (BEYER 2011: 98). A esta ambiguidade no uso e referência do termo globalização corresponde a tendência frequente de se cair na armadilha de uma análise binária opondo local e global, ou entre universal e particular (ROBERTSON 1992).

17 A leitura dos autores de referência sobre as teorias da globalização nega naturalmente as análises assentes na distinção de exclusão espacial. Pelo contrário, a globalização é considerada como um processo dialéctico entre o global e o local e a sociedade global como o resultado desse processo: «A globalização diz respeito à intersecção da presença e da ausência, ao entrelaçar de eventos sociais e de relações sociais "à distância" com as contextualidades locais» (GIDDENS 1994: 19).

18 A globalização não significa, assim, o fim do local, enquanto realidade social. O que a globalização significa de facto é uma forte e intensa conexão do local e do global, associada a um conjunto profundo de transmutações da vida quotidiana, que afectam as práticas sociais e os modos de comportamento preexistentes.

19 Na sua perspectiva culturalista da globalização, Roland Robertson (1995), defende que é um erro pensar que a globalização signifique um processo que destrua ou substitua o local ou a ideia de localidade. Mas há perspectivas diferenciadas e significativas, se não opostas, nestas leituras da relação global-local.

O que separa a leitura de Robertson da de Giddens assenta no ponto de partida: para este último, a globalização é uma consequência da modernidade enquanto para Robertson a conjunção globalização-modernidade é intrinsecamente um resultado processual e temporal. Assim, para Giddens (1994: 19) a globalização é um «fenómeno dialéctico» e a relação global-local, uma produtora de «ocorrências divergentes ou mesmo contrárias», um processo de acção-reacção; já Ronald Robertson, na sua revisitada análise da globalização, defende que a globalização «has involved there construction, in a sense of production, of "home", "community" and "locality"”.

21 Robertson defende que a relação global-local deve ser perspectivada de um modo mais subtil do que a tradicionalmente elaborada e assente na aceitação fácil de uma polaridade conflitual e em que dos polos se subsume no outro, isto é o local no global, 
mesmo quando local se refere a grandes unidades sociais como as associadas aos étniconacionalismos (ROBERTSON 1995: 26).

Para Robertson, as teorias da globalização tendem a sobrevalorizar a dimensão temporal relativamente à dimensão espacial. A utilização do paradigma da compressão do espaço-tempo - elemento nuclear da definição de globalização - tem conduzido à generalização da ideia de um imparável processo de homogeneização cultural à escala global.

23 Contrariando este quadro, Robertson (1992) sugere o uso do termo glocalização, enquanto processo em que o local e o global se entrosam para constituir o que designa por glocal. Dois aspectos são centrais na sua proposta: a noção de globalização integra a ideia de interpenetração do global e do local, ou, de um modo mais abstracto, do universal e do particularismo; que as noções contemporâneas de localidade são correntemente o produto de ideias globais, embora, como enfatiza, seja errado pensar que todas as formas de localidade sejam substantivamente homogéneas.

\section{Glocalização: 0 fim do efeito dominó}

O título deste capítulo deve-se a Zygmund Bauman (2011) e é adaptado de um texto sobre a glocalização e a compressão do mundo, isto é, sobre o efeito estruturador da compressão do tempo e do espaço. Bauman destaca com fina clareza que as distâncias geográficas deixaram de ser relevantes, isto é, obstáculos. A sua extensão deixou de ser determinante no cálculo de probabilidades. A vizinhança e a proximidade física já não são determinantes. E é por estas razões, segundo Bauman, que a metáfora do efeito dominó, assente na proximidade e na contiguidade das causas e efeitos, perdeu a sua capacidade demonstrativa.

Os estímulos, como refere Bauman, viajam independentemente das causas que lhe deram origem. Assim e destacando a interdependência e a dialéctica da relação globallocal, recorda como as causas podem ter origem local mas a sua inspiração ser global, tal como, as causas podem ter uma origem global mas os seus impactes serem/visarem o local.

Peter Beyer expressa bem esta relação entre local e global, quando afirma «the global can not be global except as a plural version of the local» (BEYER 2011: 98). Para Beyer, a noção de glocalização permite reter a ideia de que o global se expressa e se afirma pelo local enquanto este emerge como uma particularização do local.

Na sociedade da informação e do digital esta interpenetração entre local e global é naturalmente reforçada pela intensidade de links que facilitam a interconexão entre localidades e determinam a sua interdependência. Mas esta relação não facilita apenas uma eventual homogeneização de quadros e programas culturais, como funciona como factor de produção e construção de identidades locais.

Residirá no modo como é concebida a problemática da relação global-local a principal diferença entre os conceitos de modernização e de globalização. A modernização tem uma natureza exclusiva, considera vários outros, os pré-modernos e os que estão em vias de modernização. A essência do seu universalismo é essencialmente temporal, eventualmente todos poderão vir a integrar a modernidade ou as modernidades construídas.

Pelo contrário, a globalização tem uma natureza inclusiva, os seus efeitos e consequências são globais. A globalização é um conceito essencialmente espacial: «The 
global in globalization refers both to a geographic limit, the earth as a physical place, and to an encompassing range of influence, namely that all contemporary social reality is supposedly conditioned or even determined by it» (BEYER 2011: 98).

É esta realidade que os conceitos de glocalização e glocal pretendem exprimir. Ao chamarem a atenção para a complexidade da relação local-global, estes dois conceitos constituem preciosos auxiliares da análise dos processos de mudança social mas devem ser considerados como indissociáveis do conceito de globalização.

\section{Glocal: Como definir local e global?}

31 No início deste artigo referiu-se o facto de muitos dos conceitos das Ciências Sociais em uso exprimirem com dificuldade, senão com imprecisão, a realidade social actual (BECK, SLATER e RITZER 2001). Assim, o que se entende por local o vocábulo que ao longo destas páginas foi tão frequentemente referido? Tal como ele é utilizado estratégica e operacionalmente pelas grandes multinacionais, local, refere-se a grandes áreas geográficas, que podem ultrapassar as fronteiras nacionais ou referirem-se a paísescontinentes como o caso da Índia e da China, numa homogeneização cultural baseada numa ideia construída, frequentemente ideologizada, de local. A McDonalds não serve hamburgers de vaca na Índia, mas uma parte substantiva da sua população é muçulmana cujo tabu alimentar é a carne de porco e não a de vaca, esquecendo os milhões de cristãos do país que não têm este tipo de restrições.

A noção de região sofre da mesma imprecisão, sendo os seus limites definidos casuisticamente pelo utilizador. $\mathrm{O}$ uso da noção de global sofre de idênticas ambiguidades, frequentemente refere-se não a uma realidade global, isto é, à globalidade, mas apenas a uma difusão mundial. É o que se passa com muita da produção cultural ou de entretenimento, cuja aceitação é frequentemente condicionada pelas preferências locais e cujo sucesso poderá ser internacional mas não global.

Estas ambiguidades no uso de conceitos como local, regional e global não decorrem apenas da sua utilização operacional pelos agentes económicos, sendo correntes mesmo no contexto da produção científica. Ronald Robertson, por exemplo, usa o conceito de local para referir localidades concretas quer para definir realidades sociais mais vastas: "a locality has to be a standardised "form" of the local (whe the rit be a neighbourhood, a city, a country, or even a world region)» (ROBERTSON 1994: 30).

Diga-se, no entanto, que para Robertson, a noção de local e de localidade se refere quer a realidades socialmente construídas - como a construção de uma identidade ou sentimento de pertença a uma determinada comunidade - quer a realidades concretas, isto é, reificadas. Claro que esta excessiva elasticidade da escala de observação acarreta óbvias dificuldades, nomeadamente na imprecisão introduzida no quadro da investigação de pendor mais empírico. Acrescente-se que a redução de localidade a uma ideia ou sentimento contribui, ainda, para a diminuição de importância da noção de localidade como realidade concreta.

\section{A globalização do local e a localização do global}

A teoria dos sistemas sociais desde sempre destacou a dificuldade, senão impossibilidade, de um corte significativamente distintivo entre realidades sociais 
parcelares e as realidades sociais mais amplas em que se integram. Não são, assim, surpreendentes os impasses na distinção entre o local e o global. Num mundo crescentemente interconectado, as dificuldades na definição de local, frequentemente pensado como um dado adquirido, e do global, continuada e recorrentemente apresentado como um processo homogeneizador que implacavelmente asfixia a natureza singular e individual do local, são consequências expectáveis da globalização.

A proposta metodológica de Robertson constitui, por isso, um contributo essencial para a ultrapassagem desta quadratura do círculo em que muitos autores parecem ter mergulhado, num esforço ora de distinção ora de apagamento da ideia de local. Robertson, em vários dos seus trabalhos, propõe o ofuscar das fronteiras entre o local e o global e, consequentemente, o abandono das concepções teóricas assentes na ideia de acção-reacção.

Chamando a atenção para a necessidade de se deixar de considerar e analisar a globalização numa perspectiva exclusivamente, ou quase exclusivamente, macroscópica propõe que a leitura do processo de globalização envolva também os níveis mais elementares da acção social, ou seja, como designa, the real people, no seu quotidiano, interacções e mobilidade e, simultaneamente, as redes de comunidades (ROBERTSON e WHITE 2003).

Um aspecto central do pensamento de Robertson assenta na ideia de que a noção e significado de local e de localidade é o resultado de um processo de construção social. Robertson (1995: 35) salienta a invenção da ideia de localidade, assente numa ideologia do tradicional e construtora dosentimento de identidade, ${ }^{3}$ como factor fundamental na relação dialéctica estabelecida entre o global e o local.

É por esta razão que defende que a rede de interconexão estabelecida entre localidades deve ser parte constituinte da própria noção de globalização, conceptualizada como processo integrador da relação global-local, expressa enfaticamente na frase: The local has been globalized; just as the global has been localized (ROBERTSON 2003).

\section{Conclusão}

A contribuição de Ronald Robertson para uma melhor compreensão das consequências e das dinâmicas da globalização é significativa e constitui, nas suas próprias palavras, uma leitura mais subtil da articulação entre o global e o local.

41 Concebendo a globalização como um processo e definindo-a como «the compression of the world and the intensification of consciousness of the world as a whole» (ROBERTSON 1992: 8), Robertson considera como ilógicos os quadros teóricos assentes na ideia de uma tensão constante entre o local e o global, defendendo, pelo contrário, o esbater das fronteiras e propondo uma visão integradora assente nos conceitos de glocalização e de glocal, pensados como instrumentos analíticos supletivos do conceito de globalização.

O quadro teórico e conceptual que propõe constitui um precioso auxiliar para a análise dos processos de homogeneização e heterogeneização cultural. Robertson recusa a ideia de que o processo de globalização seja, como frequentemente é apresentado, um processo de homogeneização cultural ou mesmo um processo de dominação crescente da cultura de uma sociedade ou de uma região sobre as restantes.

43 O exemplo mais impressivo que apresenta é o da necessidade recorrente das grandes multinacionais em adaptarem os seus produtos às condições e tradições locais. 


\section{BIBLIOGRAFIA}

ANDERSON Benedict, 1991 (edição revista), Imagined communities. Reflections on the origin and spread of Nationalism. Londres, Verso.

BAUMAN Zygmunt, 2000, Liquid Modernity. Cambridge, Polity Press.

BAUMAN Zygmunt, 2011, «On glocalisation coming age», Social Europe Journal. [http:// www.socialeurope.eu/2011/08/on-glocalization-coming-of-age/]

BECK Ulrich, 1994, "The reinvention of politics: Towards a theory of reflexive modernization», in Ulrich Beck, Anthony Giddens e Scott Lash (eds.), Reflexive modernization. Cambridge, Polity Press, pp. 1-55.

BECK Ulrich e GRANDE Edgar, 2010, «Varieties of second modernity: the cosmopolitan turn in social and political theory and research», The British Journal of Sociology, vol. 61, n.․ 3, pp. 409-443.

BECK Ulrich e SZNAIDER Nathan, 2006, «Unpacking cosmopolitanism for the Social Sciences: a research agenda», The British Journal of Sociology, vol. 57, n.ำ 1, pp. 1-23.

BECK Ulrich; SLATER Don e RITZER George, 2001, «Interview with Ulrich Beck», Journal of Consumer Culture, vol. 1, n. 2, pp. 261-277.

BEYER Peter, 2011, «Globalization and glocalization», in James A. Beckford \& Jay Demerath, The Sage Handbook of Religions. Londres, SAGE, pp. 98-117.

Mulemba, 4 (8) | 2014 
BOUDON Raymond, 1985, La place du désordre. Critique des théories du changement social. Paris, Presses Universitaires de France.

CASTELLS Manuel, 1996, The rise of the network society. Oxford, Blackwell.

EISENSTADT Shmuel N., 2007, Múltiplas modernidades: Ensaios. Lisboa, Livros Horizonte.

FEATHERSTONE Mike e LASH Scott, 1995, «Globalization, modernity and spatialization of social theory: An introduction», in Mike Featherstone, Scott Lash e Roland Robertson (eds.), Global modernities. Londres, Sage Publications, pp. 1-24.

GELLNER Ernest, 1983, Nations and Nationalism. Oxford, Blackwell.

GIDDENS Anthony, 1987, La constitution de la société. Paris, Presses Universitaires de France.

GIDDENS Anthony, 1994, Modernidade e identidade pessoal. Oeiras, Celta Editora.

GIDDENS Anthony, 1995, As consequências da modernidade. Oeiras, Celta Editora.

HARVEY David, 1989, The condition of post modernity. An enquiry into the origin of cultural change. Oxford, Blackwell.

HOBSBAWM Eric, 1990, Nations and nationalism since 1780. Cambridge, Cambridge University Press.

KNOWLES Elizabeth e ELLIOTT Julia (eds.), 1991, The Oxford Dictionary of new words paperback. Oxford, Oxford University Press.

LIPOVETSKY Gilles, 2011, «O reino da hipercultura. Cosmopolitismo e civilização ocidental», in Gilles Lipovetsky e Hervé Juvin (eds.), o Ocidente mundializado. Controvérsia sobre a cultura planetária. Lisboa, Edições 70.

LIPOVETSKY Gilles \& SERROY Jean, 2008, La culture-monde. Réponse à une société désorientée, Paris, Odile Jacob.

LOURENÇO Nelson, 2013a, «Globalização, metropolização e insegurança: América Latina e África», Revista Direito e Segurança (Lisboa), vol. 1, n.ำ 1, pp. 87-116

LOURENÇO Nelson, 2013b, «Modernidade, globalização e nacionalismos», Mulemba - Revista Angolana de Ciências Sociais (Luanda), vol. III, n.. 6, Novembro, pp. 283-301.

ROBERTSON Roland, 1992, Globalisation. Social theory and global culture. Londres, Sage.

ROBERTSON Roland, 1994, «Globalisation or Glocalisation?», Journal of International Communication, vol. 1, n. ${ }^{\circ}$, pp. 33-52.

ROBERTSON Roland, 1995, «Glocalization: Time-space and homogeneity-heterogeneity», in Mike Featherstone, Scott Lash e Roland Robertson (eds.), Global modernities. Londres, Sage Publications, pp. 25-44.

ROBERTSON Roland, 2003, «The conceptual promise of glocalization: Commonality and

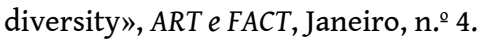

ROBERTSON Roland e WHITE Kathleen E., 2003, «Globalisation: an overview», in Roland Robertson e Kathleen E. White (eds.), Globalisation: Critical concepts in Sociology. Londres, Routledge.

SKAPSKA Grazina, 1997, «No hope? An essay on globalisation theories and the legal institution bulding processes», Droit et Société, n. 35, pp. 47-60 [«Postcommunist Europe»]. 


\section{NOTAS}

1. Anthony Giddens (1995: 52) define a globalização «como a intensificação das relações sociais à escala mundial, relações que ligam localidades distantes de tal maneira que as ocorrências locais são moldadas por acontecimentos que se dão a muitos quilómetros de distância».

2. Como propõe Raymond Boudon (1985: 25): «a noção de paradigma refere-se a um conjunto de orientações teóricas coerentes que servem de referência a um programa de investigação».

3. Ernest Gellner (1983) e Eric Hobsbawm (1990) referem as nações como uma «invenção social»; Benedict Anderson (1991) perspectiva-as como realidades «imaginadas»; sobre este assunto vide Nelson Lourenço (2013a).

4. $O$ conceito de cultura-mundo é apresentado em Gilles Lipovetsky e Jean Serroy (2008).

\section{RESUMOS}

A globalização não significa o fim do local, enquanto realidade social. Pelo contrário, a análise da modernidade deve ter presente e destacar a natureza dialéctica da globalização, enquanto processo assente na interacção do global e do local. A perspectiva culturalista da globalização mostra como é um erro pensar que a globalização significa um processo destruidor da ideia de local ou de localidade. $O$ conceito de globalização convoca a ideia de uma forte e intensa conexão do local e do global, associada às profundas transmutações da vida quotidiana, que afectam as práticas sociais e os modos de comportamento preexistentes. Os conceitos de glocal e de glocalização pretendem transmitir a necessidade de uma leitura atenta da complexidade da relação local-global, na qual a mundialização da economia e a revolução do digital desempenham um papel determinante.

Partindo do paradigma da globalização, assume-se neste artigo, que os conceitos de glocal e de glocalização constituem um quadro teórico-conceptual útil para a compreensão do funcionamento actual da economia e das dinâmicas do desenvolvimento a nível regional e local.

Globalisation does not mean the end of the Local as a social reality. Instead, the analysis of modernity must consider and highlight the dialectical nature of globalisation as a process based on the interaction of global and local. The culturalist perspective of Globalisations hows how wrong it isto think that globalisation means a destructive process of the idea of local or location. The concept of globalisation addresses the idea of a strong and intense local and global connection, associated with deep transmutations of everyday life that affect social practices and existing behaviour patterns. The concepts of glocal and glocalisation intended to convey the need for a careful reading of the complexity of local-global relationship in which economic globalisation and the digital revolution play a decisive role.

Departing from globalisation paradigm it is assumed, in this paper, that the concepts of glocal and glocalisation constitute a theoretical and conceptual framework useful for understanding the current functioning of the economy and the dynamics of development at regional and local level. 


\section{ÍNDICE}

Keywords: globalisation, glocalisation, modernity

Palavras-chave: globalização, glocalização, modernidade

\section{AUTOR}

\section{NELSON LOURENÇO}

nelson.lourenco@fcsh.unl.pt

Professor catedrático da Faculdade de Ciências Sociais e Humanas (FCSH) da Universidade Nova de Lisboa e Presidente do grupo de reflexão estratégica sobre segurança interna do Instituto de Defesa e Segurança (FD-UNL).

Doutor em Sociologia pela Universidade Nova de Lisboa (UNL), com agregação em Sociologia do Desenvolvimento e da Mudança Social pela mesma universidade, foi Professor Catedrático da Faculdade de Ciências Sociais e Humanas (FCSH-UNL); actualmente é Professor Catedrático Convidado da Faculdade de Direito da Universidade Nova de Lisboa. É Presidente do Grupo de Reflexão Estratégica sobre Segurança Interna (GRESI) e da direcção do Instituto de Direito e Segurança (FD-UNL). Publicou, entre outros, os materiais seguintes: «Globalização, metropolização e insegurança: América Latina e África», Revista de Direito e Segurança (Lisboa), vol. 1, n.. 1, 2013, pp. 87-116; «Modernidade, globalização e nacionalismos», Mulemba - Revista Angolana de Ciências Sociais (Luanda), vol. III, n.ํ 6, Novembro de 2013, pp. 283-301; «Mudança global e geopolítica dos recursos naturais», Mulemba - Revista Angolana de Ciências Sociais (Luanda), vol. III, n. 5, Maio de 2013, pp. 81-103 (com Carlos Russo Machado); «Violência urbana e sentimento de insegurança», in J. Bacelar Gouveia (coord.), Estudos de direito e segurança, volume II. Coimbra, Almedina, 2012, pp. 347-366; «Sentimento de insegurança e Estado de Direito: o espectro axial da relação de liberdade e segurança», Segurança e Defesa (Lisboa), n.. 17, AbrilJunho de 2011, pp. 70-83; «Global changes and geopolitics of natural ressources», comunicação apresentada na International conference on Ecohydrology and Climate change. Tomar, Portugal, 15-17 de Setembro de 2011; «Changement climatique et géopolitique des ressources en eau», comunicação apresentada ao encontro regional sobre Adaptation aux changements climatiques au Maghreb: Bilan et perspectives. Casablanca, Marrocos, 16-17 de Março de 2010 (com Carlos Russo Machado); «Equity, human security, and environment: key elements of soustainable development», in J. França Martins, L. Rodrigues, T. M. Fernandes (coords.), Cooperar para o desenvolvimento. Oeiras, Instituto Nacional de Administração, 2005, pp. 20-28; L. Noronha, N. Lourenço, J. P. Lobo Ferreira, A. Lleopart, E. Feoli, K. Sawkar, A. Chachadi (eds.), Coastal tourism, environment and sustainable local development. New Delhi, TERI — The Energy Research Institute, 2003. 